\title{
Weekly single agent irinotecan versus irinotecan, 5-flurouracil, and folinic acid (FOLFIRI) in advanced colorectal cancer
}

\begin{abstract}
Background and objectives: Palliative chemotherapy plays a major role in treatment of colorectal cancer (CRC) patients by improving quality of life and prolonging the survival.5-Flurouracil (FU) and Leucovorin (LV) combination were the standard of care for about 40 years. In 1990s, both oxaliplatin and irinotecan demonstrated remarkable activity against advanced $\mathrm{CRC}$ and showed survival improvement either in combination with 5-FU\LV or alone.

Patients and methods: Prospective study to compare between FOLFIRI regimen and weekly irinotecan $(125 \mathrm{mg} \backslash \mathrm{m} 2 \backslash$ week for 2 consecutive weeks followed by one week rest) as regards response, survival and toxicity profile. Results: There was no statistically significant difference between the two used protocol as regards survival and response. Toxicity profile was somewhat similar except in alopecia and infection where weekly irinotecan was less incidence of infection and more incidence of alopecia.
\end{abstract}

Conclusion: Weekly irinotecan arm is not inferior to FOLFIRI arm as regards response, survival, and toxicity profile.

Keywords: FOLFIRI, weekly irinotecan, metastatic CRC, colon, rectum
Volume 10 Issue 2 - 2019

\author{
Zedan A, Khalaf SM, Hedia A \\ Department of Medical Oncology, South Egypt Cancer Institute, \\ Assiut University, Egypt
}

Correspondence: Abdallah Hedia Mohammed at Department of Medical Oncology, South Egypt Cancer Institute, El-Methak St.,Assiut, Egypt, Email Abdallah_Elsheref@yahoo.com

Received: April 28, 2018 | Published: April 01, 2019

\section{Background}

Colorectal cancer is a common cancer worldwide. As regards the incidence, $\mathrm{CRC}$ considered the third cancer among men after prostate and lung cancer and the second among women after breast cancer. ${ }^{1}$ CRC represent about $15 \%$ of all cancers and considered the second leading cause of cancer deaths among western countries. About $50 \%$ of CRC patients developed metastatic disease despite of adjuvant therapy. ${ }^{2}$ Palliative chemotherapy plays a major role in treatment of colorectal cancer (CRC) patients by improving quality of life and prolonging the survival. 5-Flurouracil (FU) and Leucovorin (LV) combination were the standard of care for about 40 years in spite of their minimal impact on survival. ${ }^{3}$ Oxaliplatin and irinotecan demonstrate remarkable activity against advanced CRC and showed survival improvement, either alone or in combination with 5-FUlLV. ${ }^{4}$ Irinotecan is one of the topoisomerase I inhibitors. Topoisomerase I inhibition leads to double-strand DNA breaks which stimulate cell apoptosis. Addition of irinotecan to $5 \mathrm{FU} \backslash \mathrm{LV}$ significantly improves survival in advanced CRC patients. ${ }^{5}$ So, US Food and Drug Administration (FDA) approved FOLFIRI regimen as first line therapy in metastatic CRC. ${ }^{4}$ In advanced CRC patients progressed after 5-FU based therapy, weekly single agent irinotecan show promising result either by disease control or improving survival. ${ }^{6} \mathrm{We}$ conducted this study to compare the efficacy and tolerability of two irinotecan regimens (weekly irinotecan and FOLFIRI regimen) in advanced colorectal cancer.

\section{Patients and methods}

\section{Patient population:}

Between May 2013 and May 2017, a total of 52 metastatic CRC patients treated at the South Egypt Cancer Institute were recruited to participate in this study after approval of the local ethics committee and patient consent.

\section{Inclusion criteria:}

Patients of both gender, aged $\geq 18$ years with histologically confirmed colorectal adenocarcinoma; Patients with documented advanced, recurrent, or metastatic colorectal cancer according to American Joint Committee on Cancer and the International Union for Cancer Control (AJCC-UICC); 7th Edition. ECOG performance state $\leq 2$, adequate hematological, renal and hepatic functions were included in the study.

\section{Exclusion criteria:}

Patients have been treated with chemotherapy regimens containing irinotecan. Also, patients with inadequate organ functions, and serious uncontrolled concomitant disease that would contraindicate the use of any of the chemotherapy drugs or interfere with cycle's regularity were excluded from the study.

\section{Work-up:}

The routine diagnostic work-up included clinical examination, CT scans of the abdomen, pelvis within 3 weeks before starting treatment, Chest image, blood sampling for complete blood count, renal and hepatic functions. Serum level of tumor marker CEA.

\section{Treatment schedule:}

26 patients received weekly irinotecan $\left(125 \mathrm{mg} / \mathrm{m}^{2} /\right.$ week intravenous (iv) over 90 minutes for 2 consecutive weeks followed by one week rest) and the other 26 patients received FOLFIRI regimen biweekly (Irinotecan $180 \mathrm{mg} / \mathrm{m}^{2}$ intravenous day 1, Folinic acid 200 $\mathrm{mg} / \mathrm{m}^{2}$ intravenous day 1 and 2 before 5 -fluorouracil, 5-fluorouracil $400 \mathrm{mg} / \mathrm{m}^{2}$ iv bolus days 1 and 2 , then $600 \mathrm{mg} / \mathrm{m}^{2}$ iv 5-FU infusion over 22 hours, days 1 to 2). Treatment continued until disease progression, unacceptable toxicity or maximum response. Tumor response measured by the same method of assessment and same technique 
used to characterize each identified and reported lesion at baseline. Assessment was done every two cycles, accordance to the Response Evaluation Criteria in Solid Tumors (RECIST). Primary end points were overall response rate, progression free survival and side effects profile. Secondary end point was one year overall Survival.

\section{Statistical analysis}

All statistical analyses were performed using SPSS version 20.0 (SPSS Inc., Chicago, IL). Data were described as frequencies (percentages). Differences in distributions between the variables examined were analyzed by chi-square test. PFS was defined as the time from the start of treatment to the time of the first record of progression or to the date of death. OS was assessed as the time from the initiation of first-line chemotherapy to death from any cause or last follow-up. Survival curve was estimated with the KaplanMeier method and compared using the log-rank test. A multivariable Logistic regression analysis included all relevant clinicopathological variables were performed by a forward stepwise method. A P -value $<0.05$ was considered statistically significant.

\section{Results}

The demographic characteristics of the enrolled patients are shown in Table 1. Mean $( \pm \mathrm{SD})$ age of those patients received irinotecan was $45.09 \pm 12.54$ versus $47.11 \pm 10.34$ years for those received FOLFIRI. We followed the patients until May 2017 with a median follow-up period of 17 months. The tumor characteristics are shown in Table 2. Rectum was the most frequent affected site with malignancies in the current study. Ten $(38.5 \%)$ patients from irinotecan group versus $8(30.8 \%)$ patients from FOLFIRI group had rectal carcinoma. Other affected sites in the irinotecan group were sigmoid colon, ascending colon, descending colon, and transverse colon in $6(23.1 \%), 4$ $(15.4 \%), 4(15.4 \%)$ and $2(7.7 \%)$ patients respectively. Other affected sites in case of FOLFIRI were sigmoid colon, ascending colon, and descending colon in $4(15.4 \%), 4(15.4 \%)$ and $10(38.5 \%)$ patients respectively. adenocarcinoma represent the most common histological type occurred in $18(69.2 \%)$ patients in irinotecan versus 21 patients $(80.8 \%)$ in FOLFIRI group, while mucinous type occurred in 8 patients $(30.8 \%)$ in irinotecan versus 5 patients $(19.2 \%)$ in FOLFIRI group.

Table I Demographic data of studied patients

\begin{tabular}{|c|c|c|c|}
\hline Variables & $\begin{array}{l}\text { Weekly irinotecan } \\
(n=26)(\%)\end{array}$ & $\begin{array}{l}\text { FOLF IRI } \\
(n=26)(\%)\end{array}$ & P value \\
\hline Age (years) & $45.09 \pm 12.54$ & $47.11 \pm 10.34$ & 0.31 \\
\hline \multicolumn{4}{|l|}{ Sex } \\
\hline Male & $13(50)$ & I5 (57.7) & 0.39 \\
\hline Female & $13(50)$ & II (42.3) & \\
\hline \multicolumn{4}{|l|}{ Residence } \\
\hline Urban & I (3.8) & $6(23.1)$ & 0.06 \\
\hline Rural & $25(96.2)$ & $20(76.9)$ & \\
\hline \multicolumn{4}{|l|}{ Smoking } \\
\hline Non smoker & 14 (53.8) & $12(46.2)$ & \\
\hline Light smoker & 0 & $\mathrm{I}(3.8)$ & 0.73 \\
\hline Moderate smoker & $4(15.4)$ & $5(19.2)$ & \\
\hline Heavy smoker & $8(30.8)$ & $8(30.8)$ & \\
\hline
\end{tabular}

Table Continued...

\begin{tabular}{llll}
\hline Variables & $\begin{array}{l}\text { Weekly irinotecan } \\
(\mathbf{n = 2 6 )}(\%)\end{array}$ & $\begin{array}{l}\text { FOLF IRI } \\
(\mathbf{n = 2 6 )}(\%)\end{array}$ & P value \\
\hline $\begin{array}{l}\text { Response to } \\
\text { the previous } \\
\text { chemotherapy }\end{array}$ & $9(34.6)$ & $15(57.7)$ & 0.08 \\
$\begin{array}{l}\text { Sensitive } \\
\text { Resistant }\end{array}$ & I7 (65.4) & II (42.3) & \\
\hline
\end{tabular}

Continuous data was expressed in for of mean \pm SD while nominal data in form of frequency (percentage). $P$ value was significant if $<0.05$

Table 2 Tumor characteristics of the colorectal cancer in studed patients

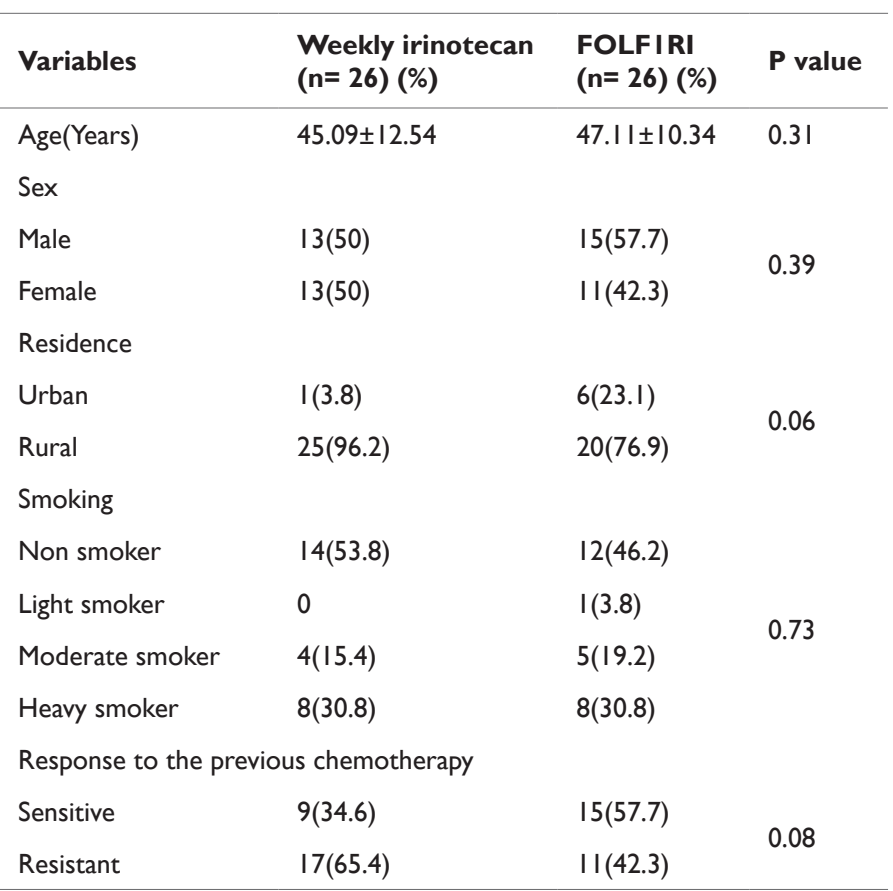

Continuous data was expressed in for of mean \pm SD while nominal data in form of frequency (percentage). $P$ value was significant if $<0.05$

By inspection of table 4, it was noticed that the toxicity profile of both groups was somewhat similar, except in infection and alopecia. Alopecia occurred in 20 patients in irinotecan group, $13(50 \%)$ patients of them were G1 while the others were G2. All patients received FOLFIRI developed alopecia, $16(61.5 \%)$ patients of them were G2 while the other were G1 which was statistically significant ( $\mathrm{P}$ value $=0.02$ ). As regard infection, Grade 1 and 2 occurred in 12 $(46.2 \%)$ and $20(76.92 \%)$ patients in irinotecan and FOLIFIRI regimen respectively while grade 3 and 4 infection occurred in only one (3.8\%) patient with irinotecan regimen and two (7.69\%) patients with FOLFIRI regimen which was statistically significant $(\mathrm{P}$ value $=0.01)$. G1 and G2 diarrhea occurred in $17(56.4 \%)$ and $13(50 \%)$ patients in irinotecan and FOLFIRI regimen respectively while G3 and G4 diarrhea occurred in $9(34.6 \%)$ and $13(50 \%)$ patients in irinotecan and FOLFIRI regimen respectively with no statistically significant difference between both group.

As regards response, in our study, partial response, stable disease and progressive disease occurred in $10(38.5 \%), 7(26.9 \%)$ and 9 $(34.6 \%)$ patients respectively in weekly irinotecan arm and $8(30.8 \%)$, $7(26.9 \%)$ and $11(42.3 \%)$ patients respectively in case of FOLFIRI regimen with no statistically significant difference between two arms (Table 3). As regards relation between the number of involved organs and response to therapy, It was noticed that number of involved 
organs in the metastasis had significant effect on response to weekly irinotecan regimen in the current study $(\mathrm{P}=0.01)$. Most of patients (71.4\%) with four organs involved in the metastasis had progressive disease while $72.7 \%$ patients with two organs involved had partial response to irinotecan. Fifty percent of those with three organs involved had stable disease. But, there is no statistically significant difference in response to FOLFIRI regimen in the current study based on number of involved organs.

Table 3 Outcome of the studied patients

\begin{tabular}{llll} 
Variables & $\begin{array}{l}\text { Weekly irinotecan } \\
(\mathbf{n = 2 6 )}\end{array}$ & $\begin{array}{l}\text { FOLFIRI } \\
(\mathbf{n}=\mathbf{2 6})\end{array}$ & P value \\
\hline Dose modification & $12(46.2)$ & $14(53.8)$ & 0.93 \\
Response & & & \\
Partial response & $10(38.5)$ & $8(30.8)$ & $0.8 \mathrm{I}$ \\
Stable response & $7(26.9)$ & $7(26.9)$ & \\
Progressive disease & $9(34.6)$ & $1 \mathrm{I}(42.3)$ & \\
Clinical benefit** & $17(65.4)$ & $15(57.7)$ & 0.09 \\
Outcome* & & & 0.65 \\
Alive & $10(38.5)$ & $12(46.2)$ & \\
Dead & $9(34.6)$ & $6(23.1)$ & \\
Unknown & $7(26.9)$ & $8(30.8)$ & \\
\hline
\end{tabular}

Data was expressed in form of frequency (percentage). $P$ value was significant if $<0.05$. Outcome was calculated till the end point of the current study $(\mathrm{I} / 5 / 2017)$. **Clinical benefit included both partial response and stable disease.

Table 4 Adverse Effects of Chemotherapy in both Groups

\begin{tabular}{|c|c|c|c|}
\hline Variables & $\begin{array}{l}\text { Weekly irinotecan } \\
(n=26)\end{array}$ & $\begin{array}{l}\text { FOLFIRI } \\
(n=26)\end{array}$ & $P$ value \\
\hline \multicolumn{4}{|c|}{ Nausea \& vomiting } \\
\hline GI,2 & $22(84.6)$ & $21(80.8)$ & 0.13 \\
\hline $\mathrm{G} 3,4$ & $4(15.4)$ & $5(19.2)$ & \\
\hline Diarrhea & & & 0.71 \\
\hline GI,2 & $20(76.93)$ & $19(69.3)$ & \\
\hline $\mathrm{G} 3,4$ & $6(23.7)$ & $8(30.7)$ & \\
\hline Mucositis & & & 0.63 \\
\hline GI,2 & $19(73.1)$ & $18(69.2)$ & \\
\hline $\mathrm{G} 3,4$ & $7(26.9)$ & $8(30.8)$ & \\
\hline Fever & & & 0.46 \\
\hline No & $6(23.1)$ & $4(15.4)$ & \\
\hline GI,2 & $20(76.9)$ & $22(84.6)$ & \\
\hline Hepatoxicity & & & 0.67 \\
\hline No & $15(57.7)$ & $20(76.92)$ & \\
\hline $\mathrm{GI}, 2$ & $7(26.9)$ & $4(I 5.38)$ & \\
\hline $\mathrm{G} 3,4$ & $4(15.4)$ & $2(7.69)$ & \\
\hline Bleeding & & & 0.36 \\
\hline No & $21(80.8)$ & $21(80.8)$ & \\
\hline GI,2 & $\mathrm{I}(3.8)$ & $4(15.4)$ & \\
\hline $\mathrm{G} 3,4$ & $4(15.4)$ & $\mathrm{I}(3.8)$ & \\
\hline
\end{tabular}

Table Continued...

\begin{tabular}{|c|c|c|c|}
\hline Variables & $\begin{array}{l}\text { Weekly irinotecan } \\
(n=26)\end{array}$ & $\begin{array}{l}\text { FOLFIRI } \\
(n=26)\end{array}$ & $P$ value \\
\hline Infection & & & 0.01 \\
\hline No & $12(50)$ & $4(15.38)$ & \\
\hline GI,2 & $12(46.2)$ & $20(76.92($ & \\
\hline $\mathrm{G} 3,4$ & $\mathrm{I}(3.8)$ & $2(7.69)$ & \\
\hline Alopecia & & & 0.02 \\
\hline No & $6(23.1)$ & 0 & \\
\hline GI,2 & $13(50)$ & $10(38.5)$ & \\
\hline G3,4 & $7(26.9)$ & $16(6 \mid .5)$ & \\
\hline Neutropenia & & & 0.63 \\
\hline No & $3(11.5)$ & $4(15.4)$ & \\
\hline GI,2 & $22(84.6)$ & $19(73.1)$ & \\
\hline $\mathrm{G} 3,4$ & $\mathrm{I}(3.8)$ & $3(\mid I .5)$ & \\
\hline Thrombocytopenia & & & 0.06 \\
\hline No & $4(15.4)$ & 0 & \\
\hline GI,2 & $15(69.2)$ & $25(96.2)$ & \\
\hline$G 3,4$ & $4(15.4)$ & I(3.8) & \\
\hline
\end{tabular}

Data was expressed in form of frequency (percentage). P value was significant if $<0.05$

As regards survival, in our study Kaplan Meier survival analysis was used to estimate the overall survival of both groups included in the study. Median survival of all patients included in the study was 10.15 months while median survival of patients who received weekly irinotecan regimen was 10 months versus 8.77 months for those received FOLFIRI regimen with no statistically significant difference between two regimens $(\mathrm{P}=0.86)$. One year survival for weekly irinotecan regimen was $37.4 \%$ versus $36.3 \%$ for FOLFIRI regimen (Figure 1). In our study, it was noticed that 19 (73.08\%) patients received FOLFIRI regimen had progressive disease and median time to disease progression was 11 months while $22(84.6 \%)$ patients had progressive disease in case of irinotecan regimen with median time to disease progression was 8.8 months, with no statistically significant difference $(\mathrm{P}=0.56)$ (Figure 2).

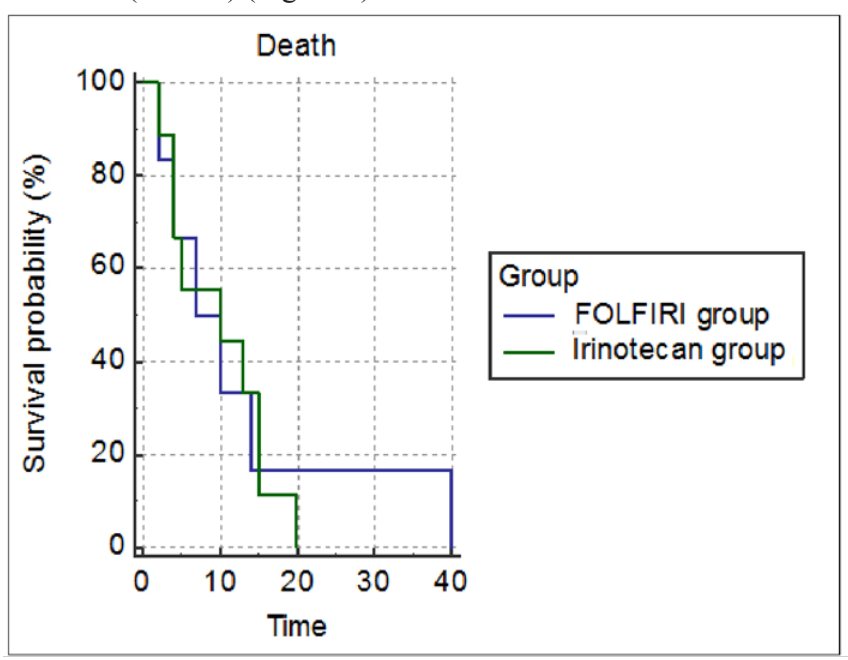

Figure I Kaplan Meier Survival Analysis in the Study. 


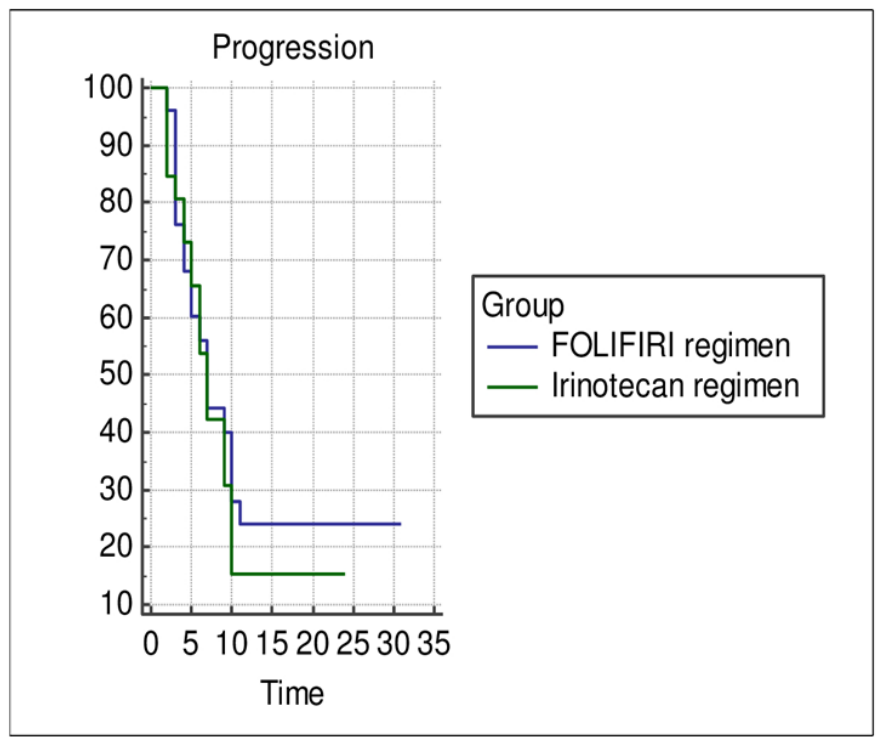

Figure 2 Progression free survival in both regimens.

\section{Discussion}

Colorectal cancer is a common cancer worldwide. As regards the incidence, $\mathrm{CRC}$ considered the third cancer among men after prostate and lung cancer and the second among women after breast cancer. Palliative chemotherapy plays a major role in treatment of colorectal cancer (CRC) patients by improving quality of life and prolonging the survival. 5-Flurouracil (FU) and Leucovorin (LV) combination were the standard of care for about 40 years in spite of their minimal impact on survival. ${ }^{3}$ Addition of irinotecan to 5 FUlLV significantly improves survival in advanced CRC patients. ${ }^{5}$ So, US Food and Drug Administration (FDA) approved FOLFIRI regimen as first line therapy in metastatic CRC. ${ }^{4}$ In advanced CRC patients progressed after 5-FU based therapy, weekly single agent irinotecan show promising result either by disease control or improving survival. ${ }^{6} \mathrm{We}$ conducted this study to compare the efficacy and tolerability of two irinotecan regimens (weekly irinotecan and FOLFIRI regimen) in advanced colorectal cancer.

In our study, the mean age $( \pm \mathrm{SD})$ of our patients who received weekly irinotecan was $45.09 \pm 12.5$ years while the mean age of those patients who received FOLFIRI regimen was $47.11 \pm 10.34$ years. About $34.6 \%$ of them below the age of 40 years, these results are similar to data observed in Abou-Zeid et al., ${ }^{7}$ in which incidence of $\mathrm{CRC}$ in patient below age of 40 years ranged from $35.6 \%$ to $38 \%$. These data are also similar to a study done by Veruttipong et al., ${ }^{8}$ who said that, the incidence rate of CRC in Egypt under the age of 40 years is slightly higher than the United States. Veruttipong et al., explained the high young-onset rate may be due to westernization of the lifestyle or due to intense environmental exposures.

As regards non-hematological toxicity, in our study, grade I-Il nausea and vomiting occurred in $22(84.6 \%)$ patients who received weekly irinotecan and occurred in $21(80.8 \%)$ patients who received FOLFIRI regimen. While grade III-IV occurred in $4(15.4 \%)$ patients in weekly irinotecan group and 5 (19.2\%) patients of FOLFIRI group with no statistically significant difference between two groups ( $P$ value $=0.13$ ). These data are in contrary with data shown in Clark et al., ${ }^{9}$ where grade III-IV vomiting occurred more common with single agent irinotecan (19\%) than with FOLFIRI regimen (10\%). In our study, Grade III-IV diarrhea occurred in $6(23.07 \%)$ patients in the weekly irinotecan arm and $8(30.7 \%)$ patients in the FOLFIRI arm with no statistically significant difference between two arms (P value $=0.71$ ). Consistent with our results, Fuchs et al., ${ }^{10}$ demonstrated that grade III-IV diarrhea occurred in $36 \%$ of patients received weekly irinotecan.

As regards hematological toxicity, in our study, grade I-II anemia occurred in $8(30.76 \%)$ patients who received weekly irinotecan and $14(53.84 \%)$ patients who received FOLFIRI regimen while, grade III-IV anemia occurred in four $(15.38 \%)$ patients who received weekly irinotecan and five (19.23\%) patients who received FOLFIRI regimen with no statistically significant difference between two groups $(\mathrm{P}$ value $=0.32)$. In contrary, Rosati et al., ${ }^{6}$ demonstrated that grade I-II anemia occurred in $78 \%$ while grade III-IV occurred in $8.6 \%$ of patients received weekly irinotecan. Grade III-IV neutropenia occurred in only one patient (3.8\%) of weekly irinotecan arm and in three patients (11.5\%) of FOLFIRI arm with no statistically significant difference between two arms ( $\mathrm{P}$ value $=0.63$ ). Comparable results have been reported by Clarke et al., ${ }^{9}$ where grade III-IV neutropenia occurred in 5\% of patients in weekly arm and 14\% of FOLFIRI arm.

As regards infection, Grade I-II infection occurred in 12 (46.2\%) patients in weekly irinotecan arm and $20(76.92 \%)$ patients of FOLFIRI arm while grade III-IV occurred only in one (3.8\%) patient in weekly irinotecan arm and two $(7.69 \%)$ patients in FOLFIRI arm which was statistically significant $(\mathrm{P}$ value $=0.01$ ). These data are consistent with data shown in Schoemaker et al., ${ }^{3}$ where grade III-IV infection occurred in $5 \%$ of patients who received weekly irinotecan.

As regards alopecia, in our study, grade I-II occurred in $13(50 \%)$ patients in weekly irinotecan arm and $10(38.5 \%)$ patients with FOLFIRI arm which was statistically significant $(\mathrm{P}$ value $=0.02)$. These data are somewhat similar to data observed in Rosati et al., ${ }^{6}$ where grade I-II alopecia occurred in $47.7 \%$ of patients received weekly irinotecan. As regards response, in our study, partial response, stable disease and progressive disease occurred in $10(38.5 \%), 7$ $(26.9 \%)$ and $9(34.6 \%)$ patients respectively in weekly irinotecan arm and $8(30.8 \%), 7(26.9 \%)$, and $11(42.3 \%)$ patients respectively in case of FOLFIRI regimen with no statistically significant difference between two arms. These data are in contrary with data observed in Schoemaker et al., ${ }^{3}$ where partial response, stable disease and progressive disease occurred in 5\%,62\% and $19 \%$ in patient received weekly irinotecan respectively. It was noticed that number of involved organs in the metastasis had significant effect on response to weekly irinotecan regimen in the current study $(\mathrm{P}=0.01)$. Most of patients $(71.4 \%)$ with four organs involved in the metastasis had progressive disease while $72.7 \%$ patients with two organs involved had partial response to irinotecan. Fifty percent of those with three organs involved had stable disease. But, there is no statistically significant difference in response to FOLFIRI regimen in the current study based on number of involved organs. These data are somewhat similar to data observed at Saltz et al., ${ }^{5}$ where combination regimen was better response rate than single agent regimen in patients with $>2$ site of metastasis.

As regards survival, in our study Kaplan Meier survival analysis was used to estimate the overall survival of both groups included in the study. Median survival of all patients included in the study was 10.15 months while median survival of patients who received weekly irinotecan regimen was 10 months versus 8.77 months for those received FOLFIRI regimen with no statistically significant difference 
between two regimens (P value=0.86). One year survival for weekly irinotecan regimen was $37.4 \%$ versus $36.3 \%$ for FOLFIRI regimen. As regards weekly irinotecan arm, these data are somewhat similar to data shown in Saltz et al., ${ }^{5}$ where median survival in patients who received weekly irinotecan was 11 months. As regards FOLFIRI arm, our data are in contrary with data shown in Colucci et al., ${ }^{11}$ where median survival in patients received FOLFIRI was 14 months.

In our study, it was noticed that $19(73.08 \%)$ patients received FOLFIRI regimen had progressive disease and median time to disease progression was 11 months while $22(84.6 \%)$ patients had progressive disease in case of irinotecan regimen with median time to disease progression was 8.8 months, with no statistically significant difference $(\mathrm{P}$ value $=0.56)$. These data are in contrary with data shown in Saltz et al., ${ }^{5}$ where median TTP was 4.2 months in weekly single agent irinotecan and 7 months in combination arm.

\section{Conclusion}

In summary, Our findings concluded that, weekly irinotecan arm not inferior to FOLFIRI arm as regards response, survival, and toxicity profile.

\section{Recommendation}

Larger number of patients with longer period of follow up will be required to confirm the results. Weekly irinotecan shouldn't be offered to heavily metastatic colorectal cancer patients with 4 or more involved organs.

\section{Conflicts of interest}

We declare that we don't have any conflict of interest.

It is the first time to submit this article.

\section{References}

1. Jemal A, Ward EM, Johnson CJ. Annual Report to the Nation on the Status of Cancer, 1975-2014, Featuring Survival. J Natl Cancer Inst. 2017;109(9).
2. Ahmed G, Basel E, Aida A. Colorectal cancer in Egypt is commoner in young people: Is this cause for alarm? Alexandria Journal of Medicine. 2014;50(3):197-201.

3. Schoemaker N, Kuppens I, Moiseyenko V. A randomised phase II multicentre trial of irinotecan (CPT-11) using four different schedules in patients with metastatic colorectal cancer. Br J Cancer. 2004;91(8):14341441.

4. Douillard JY, Cunningham D, Roth AD. Irinotecan combined with fluorouracil compared with fluorouracil alone as first-line treatment for metastatic colorectal cancer; A multicentre randomised trial. The Lancet. 2000;355(92.9):1041-1047.

5. Saltz LB, Cox JV, Blanke C. Irinotecan plus fluorouracil and leucovorin for metastatic colorectal cancer. $N$ Engl J Med. 2000; 343(13):905-914.

6. Rosati G, Cordio S. Single-agent irinotecan as second-line weekly chemotherapy in advanced colorectal cancer. Tumori. 2006; 92(4):290294.

7. Abou-zeid AA, Khafagy W, Marzouk DM. Colorectal Cancer in Egypt. Dis Colon Rectum. 2002;45(9):1255-1260.

8. Veruttipong D, Soliman AS, Gilbert SF, et al. Age distribution, polyps and rectal cancer in the Egyptian population-based cancer registry. World J Gastroenterol. 2012;18(30):3997-4003.

9. Clarke S, Yip S, Brown C, et al. Single-agent irinotecan or FOLFIRI as second-line chemotherapy for advanced colorectal cancer; results of a randomised phase II study (DaVINCI) and meta-analysis. Eur J Cancer. 2012;47 47(12):1826-1836.

10. Fuchs CS, Moore MR, Harker G: Phase III comparison of two irinotecan dosing regimens in second-line therapy of metastatic colorectal cancer. $J$ Clin Onco. 2003;21(5):807-814.

11. Colucci G, Gebbia V, Paoletti G, et al. Phase III randomized trial of FOLFIRI versus FOLFOX4 in the treatment of advanced colorectal cancer: a multicenter study of the Gruppo Oncologico Dell'Italia Meridionale. J Clin Oncol. 2005;23(22):4866-4875. 